LUNG CANCER

\title{
Ultrasound guided cytological aspiration of supraclavicular lymph nodes in patients with suspected lung cancer
}

\author{
M Kumaran, R E Benamore, R Vaidhyanath, S Muller, C J Richards, M D Peake, J J Entwisle
}

Thorax 2005;60:229-233. doi: 10.1136/thx.2004.029223

See end of article for authors' affiliations

......................

Correspondence to:

Dr R E Benamore

Glenfield Hospital, Groby

Road, Leicester LE3 9QP,

UK; rachelbenamore@

doctors.org.uk

Received 31 May 2004

Accepted

12 November 2004

\begin{abstract}
Background: Lung cancer is the leading cause of death from cancer in the UK. Pathological diagnosis traditionally requires invasive procedures such as bronchoscopy, mediastinoscopy, or image guided biopsy. Ultrasound of the neck with fine needle aspiration cytology (FNAC) of enlarged but impalpable supraclavicular nodes has been used in patients with suspected lung cancer who have N2 or N3 disease on staging computed tomography (CT). If positive, this technique helps to both stage the patient and provide a cytological diagnosis.

Methods: 101 patients were enrolled prospectively over a 1 year period. FNAC was performed on all supraclavicular nodes over $5 \mathrm{~mm}$ in size using the capillary aspiration technique.

Results: Sixty one of the 101 patients had enlarged supraclavicular nodes and underwent FNAC. The overall malignant yield was $45.5 \%$ of patients scanned and $75.4 \%$ of patients sampled. As a result of FNAC, 43 patients $(42.6 \%)$ avoided more invasive procedures.

Conclusion: Ultrasound guided FNAC is a promising, relatively non-invasive technique for the staging and diagnosis of patients with lung cancer.
\end{abstract}

$\mathrm{L}$ ung cancer is a common disease with over 38410 new cases diagnosed in the UK in 2000. Recent 2002 statistics -revealed that lung cancer accounts for 33600 deaths per year, representing $22 \%$ of all cancer related deaths in the UK. It is estimated that, of those patients with newly diagnosed lung cancer, $26 \%$ will have involved mediastinal nodes and $49 \%$ distant metastases at presentation. ${ }^{1}$ Patients being considered for palliative and radical treatment need both accurate staging and cell typing as prognosis and treatment are related to both. Survival rates for advanced lung cancer are poor, being $8.4 \%$ for stage III disease and only $1.6 \%$ for stage IV. ${ }^{2}$ A rapid relatively non-invasive test would therefore be beneficial.

Supraclavicular lymph node involvement is considered N3 disease according to the TNM classification. ${ }^{3}$ Cytological aspiration of palpable nodes is a well established technique. ${ }^{4}$ Historically, there has been knowledge of occult supraclavicular lymph node involvement which may be sampled surgically. ${ }^{5}$

Lymphadenopathy in the supraclavicular region can be difficult to detect on clinical examination. The sensitivity of ultrasound for detecting nodes of more than $1 \mathrm{~cm}$ is $16.7 \%$ higher than clinical examination. ${ }^{6}$ When compared with histological examination following surgical excision, the sensitivity and specificity of supraclavicular ultrasound is $91 \%$ and $94 \%$ compared with $82 \%$ and $83 \%$ for clinical palpation and, importantly, ultrasound detects up to $50 \%$ of occult non-palpable metastases. ${ }^{7}$ Ultrasound with fine needle aspiration cytology (FNAC) has been well described for cancer of the head and neck with sensitivity and specificity for malignancy of over $90 \% .^{8}$ In patients with a known malignancy and suspicion of neck node involvement, nodes as small as $4 \mathrm{~mm}$ can contain metastases. ${ }^{9}$ Sonographically the nodes are detected easily; malignant nodes become rounded with an increased short axis diameter and develop a heterogeneous echo texture. ${ }^{10}$ On colour or power Doppler these nodes lose their normal central (hilar) vasculature and develop a predominantly peripheral (capsular) flow pattern. ${ }^{11}{ }^{12}$ However, these features are not specific or sensitive for malignant infiltration. ${ }^{13}$
Recently published literature has suggested that ultrasound guided FNAC is an effective way of identifying and sampling non-palpable malignant supraclavicular nodes in patients with suspected lung cancer. ${ }^{14}{ }^{15}$ Fultz et al ${ }^{14}$ studied 55 patients without palpable supraclavicular nodes; 17 (31\%) had supraclavicular metastases but $46 \%$ of patients with enlarged mediastinal nodes on the CT scan had supraclavicular node involvement. In our experience it is unusual for disease to involve supraclavicular nodes in the absence of enlarged mediastinal nodes on the CT scan. Our hypothesis is that, in patients with enlarged mediastinal nodes on staging CT, we could expect a higher diagnostic yield from supraclavicular ultrasound and FNAC than scanning all patients with suspected lung cancer. We therefore hypothesised that, in this group of patients, supraclavicular node FNAC provides an easy method of obtaining both a cytological diagnosis and nodal stage, and reduces the need for more invasive procedures.

\section{METHODS}

Over a 12 month period from 1 April 2003, 101 patients (64 men) of median age 69.5 years (range 43-93) were prospectively recruited. All patients with suspected lung cancer, with non-palpable supraclavicular nodes and with enlarged mediastinal nodes seen on the CT scan were enrolled in the study. The CT criterion for enlarged nodes was a short axis diameter of more than $1 \mathrm{~cm}$. The median delay between staging CT scan and ultrasound was 5 days (range 0-53). Ultrasound of the neck and supraclavicular region was performed using a $7-12 \mathrm{MHz}$ linear probe on an ATL 5000 HDI machine. Enlarged nodes on ultrasound were defined as having a short axis diameter of more than $5 \mathrm{~mm}$. Node shape and blood flow pattern were documented. A cut off size of $5 \mathrm{~mm}$ is reasonable as it is often technically impossible to sample smaller nodes.

After obtaining informed verbal consent, ultrasound guided FNAC of enlarged nodes was performed using the capillary aspiration technique. The patients were placed supine with the neck hyperextended by placing a small pillow under the shoulders. This position was not possible in 
Table 1 Antibodies used for immunocytochemistry

\begin{tabular}{llll}
\hline Antibody & Supplier & Pretreatment & Dilution \\
\hline BER EP4 & Dako & & $1: 300$ \\
CD 56 & Novocastra & MW 12 min & $1: 50$ \\
CD 45 & Dako & MW 12 min & $1: 50$ \\
Synaptophysin & Dako & & $1: 25$ \\
Chromogranin & Dako & MW 12 min & $1: 50$ \\
CD 30 & Dako & & $1: 10$ \\
CAM 5.2 & Beckton- & & $1: 20$ \\
& Dickinson & & $1: 50$ \\
AE1/3 & Dako & MW 12 min & $1: 50$ \\
HMB 45 & Dako & & $1: 25$ \\
CK 7 & Dako & & $1: 25$ \\
CK 20 & Dako & & $1: 50$ \\
TF & Dako & & $1: 5000$ \\
TG & Dako & PC citrate buffer & $1: 40$ \\
ER & Novocastra & pH 6 for 6.5 min & \\
& & \multicolumn{2}{l}{}
\end{tabular}

all patients due to the effects of the lung cancer and associated respiratory co-morbidity and the technique was performed in the erect position in these cases. Local anaesthesia was not required. Enlarged nodes were aspirated with a fine 21 gauge needle, the needle being rotated during the sampling. At least two passes were made. Unlike other aspiration techniques, a syringe and suction was not used. The aspirated material contained within the needle or its hub was injected into liquid cytological preparation solution. Four principal operators - two chest radiologists and two head and neck radiologists-performed the procedures. The capillary technique of FNAC was used in all cases.

The aspirates were sent to the pathology department in cytorich red cytospin fluid. No direct spreads for rapid reporting were prepared. Two cytospin preparations were prepared from each sample and were stained using the Papanicolau stain and haematoxylin and eosin. The preparations were looked at independently by two experienced cellular pathologists. In nine cases where interpretation was not straightforward, immunocytochemistry (ICC) was also carried out. Details of the antibodies used for ICC are given in table 1 .

Fine needle aspiration of palpable lymphadenopathy has been a long standing practice in our centre, so formal ethical approval was not sought.

\section{RESULTS}

One hundred and one patients were enrolled in the study, 62 of whom had enlarged supraclavicular nodes on ultrasound (mean $13.3 \mathrm{~mm}$, range 5-30). One patient had a $5 \mathrm{~mm}$ supraclavicular node which was not technically possible to sample, so 61 patients $(60.4 \%)$ underwent FNAC. A positive malignant cytological result was obtained initially in 44 patients $(72.1 \%$ of those sampled). Two patients with initial negative results but with suspicious ultrasound features were recalled for a repeat aspirate and both yielded a malignant

Table 2 Malignant and suspicious cytological diagnoses obtained by fine needle aspiration

\begin{tabular}{lc}
\hline Cell type & No of patients \\
\hline Non-small cell cancer (NSCLC) & 33 \\
Small cell cancer (SCLC) & 8 \\
Lymphoma & 1 \\
Suspicious for lymphoma & 3 \\
Metastatic breast cancer & 1 \\
Malignant (uncertain cell type) & 3 \\
\hline
\end{tabular}

result subsequently. The overall malignant yield was therefore 46 of 101 patients scanned (45.5\%) and 46 of 61 patients sampled $(75.4 \%)$. As a gold standard of formal surgical neck exploration was not performed in the study population, we are unable to provide true sensitivity and specificity values for the technique. However, in the 88 patients with a final diagnosis of lung cancer, $44(50 \%)$ had a malignant FNAC.

Immunocytochemistry was performed on nine FNAC samples. In six cases ICC clarified the tumour type (four non-small cell carcinoma (NSCLC), one small cell carcinoma (SCLC), one high grade lymphoma) and in three cases the cell type (SCLC versus NSCLC) was still uncertain following ICC.

Table 2 shows the range of malignant and suspicious cytological diagnoses obtained at FNAC. All 101 patients were suspected of having lung cancer and had enlarged N2 nodes at staging CT. 62 had enlarged supraclavicular nodes at ultrasound, of which 61 were sampled. Of these 61 patients sampled, 46 were malignant (including two patients who underwent repeat FNAC), three were suspicious for lymphoma, eight were negative, and six were acellular (two of these patients underwent repeat FNAC).

Four patients had an eventual diagnosis of lymphoma; one had a malignant FNAC and three aspirates were reported as "suspicious of lymphoma" and histological confirmation was advised. The patient with the "malignant" FNAC died before formal node excision could be performed, but ICC on the FNA sample supported a diagnosis of lymphoma. Of the three "suspicious" cases, two had an eventual histological diagnosis made by formal lymph node biopsy and one by bronchoscopy. Of the patients with uncertain malignant cell type, one was a clinical diagnosis of SCLC as the patient was treated palliatively. One patient underwent CT guided lung biopsy with an eventual diagnosis of NSCLC. One patient had a repeat lymph node FNAC which was non-contributory and, as the patient was too unwell for further investigations, a clinical diagnosis of lung cancer was made. By using FNAC, a total of 43 patients avoided more invasive diagnostic procedures.

Table 3 shows details of the patients with an eventual diagnosis of SCLC and NSCLC (including those with a clinical diagnosis who were too unwell for treatment). Stage group and details of enlarged nodes and FNAC results are shown. Six samples were acellular. FNAC was repeated in two patients because, as described above, the ultrasound features of the enlarged nodes were suspicious for malignant infiltration. The repeat FNAC yielded a positive malignant result in both. One patient was too unwell for further investigations and a clinical diagnosis of lung cancer was made. One patient had a surgical lymph node excision (NSCLC) as the node was technically difficult to access by ultrasound. The ultrasound features of the enlarged nodes in the other two patients were equivocal so alternative methods of diagnosis were used: one had an ultrasound guided lung biopsy (NSCLC) and one patient underwent bronchoscopy (NSCLC).

The final diagnoses for all 101 patients are shown in table 4 . One patient had mediastinoscopy and thymic resection for tumour which, after expert opinion, was considered to represent a well differentiated sclerosing liposarcoma. Of the four patients with a benign diagnosis, all cases were followed up by repeat CT scanning which showed resolution of the abnormalities so a retrospective diagnosis of infection was made. FNAC did not identify a specific benign diagnosis in the two patients with enlarged supraclavicular nodes.

Of the 77 patients with proven or suspected NSCLC, 27 patients had N2 disease without metastases, 17 had N3 disease without metastases, and 33 had metastases on 
Table 3 Characteristics of patients with eventual diagnosis of NSCLC or SCLC

\begin{tabular}{|c|c|c|c|c|c|c|}
\hline \multirow[b]{2}{*}{ Cell type } & \multirow[b]{2}{*}{ CT stage } & \multirow[b]{2}{*}{ No of patients } & \multicolumn{2}{|c|}{ Enlarged nodes at US? } & \multicolumn{2}{|l|}{ FNAC result } \\
\hline & & & Yes & No & Positive & Negative \\
\hline \multirow[t]{3}{*}{ NSCLC } & IIIA & 18 & 10 & 8 & 6 & $4(2 s, 2 u)$ \\
\hline & IIIB & 26 & 14 & 12 & $11(1$ ?cell type) & $3(2 s, 1 u)$ \\
\hline & IV & 33 & 21 & 12 & 18 (1 ?cell type) & $3(1 \mathrm{~s}, 1 \mathrm{v}, 1$ not sampled) \\
\hline \multirow[t]{2}{*}{$\mathrm{SCLC}$} & Limited & 7 & 6 & 1 & 6 (1 ?cell type) & 0 \\
\hline & Extensive & 4 & 3 & 1 & 3 & 0 \\
\hline
\end{tabular}

NSCLC, non-small cell lung cancer; SCLC, small cell lung cancer; US, ultrasound; FNAC, fine needle aspiration cytology; s= satisfactory sample; u= unsatisfactory sample.

staging CT. Nine patients were upstaged from N2M0 to N3M0 as the result of a positive FNAC.

\section{DISCUSSION}

Contrast enhanced CT scanning of the thorax and abdomen is normally the initial staging investigation but has significant limitations in mediastinal nodal assessment. The standard criterion for mediastinal nodal enlargement on CT scans is considered to be $10 \mathrm{~mm}$ in short axis diameter, ${ }^{16}$ but there is poor correlation between CT nodal size and malignant infiltration. ${ }^{17} \mathrm{~A}$ recent meta-analysis of 39 studies showed that CT scanning had a median sensitivity and specificity for detecting lymph node involvement of $61 \%$ and $79 \%$, respectively. Positron emission tomography (PET) had a significantly improved median sensitivity and specificity with values of $85 \%$ and $90 \%$, respectively, and, in addition, it may detect other occult distant metastases. ${ }^{18}$ However, this is not routinely available in most areas of the UK.

CT scanning relies on anatomical size and PET on metabolic activity, but neither technique confirms the pathological diagnosis and selected patients may need mediastinal nodal sampling. This can be achieved by CT biopsy, endoscopic ultrasound, transbronchial needle biopsy, and a number of surgical techniques. In patients with metastatic disease being considered for chemotherapy, a tissue diagnosis is usually obtained by percutaneous biopsy or bronchoscopy. The most common complications of CT biopsy are pneumothorax and haemorrhage. ${ }^{19}$

On CT scanning the supraclavicular nodes are above the manubrium and in close proximity to the clavicles. On ultrasound these groups are at the angle of the jugular and subclavian veins. They are also described as scalene nodes in view of their proximity to this muscle group. In TNM staging of NSCLC the supraclavicular lymph nodes, if involved, are considered $\mathrm{N} 3$ disease. Lymphadenopathy above this level at the lower jugular chain is considered Ml disease. ${ }^{3}$
There have been reports of supraclavicular node sampling in cases of both lung and gastrointestinal malignancy. Previous experience of surgical biopsy of non-palpable supraclavicular nodes in patients with bronchogenic carcinoma did not yield encouraging results, with only $8.9 \%$ showing nodal metastatic disease..$^{20}$ However, the sensitivity of ultrasound for detecting nodes greater than $1 \mathrm{~cm}$ is $16.7 \%$ higher than clinical examination. ${ }^{6}$ When compared with histology from surgical excision, the sensitivity and specificity of supraclavicular ultrasound is $91 \%$ and $94 \%$ versus $82 \%$ and $83 \%$ for clinical palpation and, importantly, ultrasound detects up to $50 \%$ of occult non-palpable metastases. ${ }^{7}$

Ultrasound with FNAC is well described in the literature on head and neck cancer with sensitivity and specificity for malignancy of over $90 \% .{ }^{8}$ Sonographically the nodes are detected easily and the features of malignant infiltration are described. ${ }^{10}$ Malignant nodes become rounded with loss of the normal fatty hilum and a predominantly capsular flow on colour or power Doppler. ${ }^{11} 12$ These features are not specific or sensitive for malignant infiltration ${ }^{13}$ and, in view of this, we based our criteria purely on size. Capsular flow and heterogeneity, if demonstrated, may be helpful in determining which patients to recall if the initial cytological examination is unsuccessful. We recalled two patients as their initial samples were inadequate but whose ultrasound demonstrated these features. Repeat FNAC in these cases yielded malignant results. The other patients with inadequate FNAC underwent alternative diagnostic procedures as ultrasound features were not suspicious.

The capillary aspiration technique is simple and safe and is well described in the head and neck literature. ${ }^{21}$ The technique relies on the fact that the negative pressure applied by suction has a relatively minor role compared with the cutting or scraping action of the bevelled needle. Unlike other aspiration techniques, a syringe and suction are not used. The aspirated material usually contained within the needle or its

Table 4 Final diagnoses for all patients $(n=101)$

\begin{tabular}{lllll}
\hline Final diagnosis & $\begin{array}{l}\text { No confirmed } \\
\text { pathologically }\end{array}$ & $\begin{array}{l}\text { No of clinical } \\
\text { diagnoses }\end{array}$ & Total no (\%) & $\begin{array}{l}\text { No where diagnosis } \\
\text { suggested/confirmed by } \\
\text { FNAC (\%) }\end{array}$ \\
\hline $\begin{array}{l}\text { Lung cancer } \\
\text { NSCLC }\end{array}$ & 78 & 10 & $88(87.1 \%)$ & $44(50)$ \\
suspicious/negative FNAC
\end{tabular}


hub can be applied to glass slides and spread or, as in our centre, injected into liquid cytological preparation solution. In our series no patient refused the sampling technique and there were no known complications. Two patients with a new diagnosis of suspected lung cancer had clinical evidence of superior vena cava obstruction. Concern has been expressed regarding the increased risk of bleeding following biopsy in this condition. A rapid cytological diagnosis without complications was made by ultrasound guided FNAC. This has been reported as a safe technique in patients with superior vena cava obstruction and palpable neck lymphadenopathy. ${ }^{22}$ The calibre of the sampling needle is smaller than those used in most biopsy techniques. Neck node sampling has the added advantage that direct pressure can be applied to the sampling site. Three patients who presented with recent venous thromboembolism were sampled while anticoagulated. A positive malignant cytological diagnosis was made in all cases and there were no bleeding complications.

Because this technique is relatively non-invasive, it could potentially be used in all patients with suspected lung cancer. In view of the reported association between CT mediastinal adenopathy and supraclavicular metastases ${ }^{14}$ and because of limited resources, we chose this select patient group. CT scanning of the supraclavicular region is difficult to evaluate because of the complex anatomy. Many of these nodes were not identified prospectively on the CT scan but this area should be carefully examined. We now include the lower neck on the initial staging scan. If supraclavicular nodes are detected on CT scanning, ultrasound and FNAC sampling should be performed.

In our centre there is no provision for immediate on site cytological review which may improve diagnostic yield. ${ }^{23}$ The cytological interpretation is generally straightforward, although it is not always possible to assign cell type accurately, even if ICC is also carried out on the FNAC. Core biopsy using small gauge cutting needles is a safe technique and can be performed in an outpatient setting. ${ }^{24}$ This technique may be helpful in determining cell type in indeterminate FNAC cases or in patients with suspected lymphoma.

Of the patients scanned, the overall yield for malignancy was $45.5 \%$ and for carcinoma was $44.6 \%$, although two of the patients required further sampling. This is not a true representation of the incidence of supraclavicular nodes as there is a strong inclusion bias. The authors feel this is an acceptable yield and is higher than other reported series where there were broader inclusion criteria. Fultz et al ${ }^{14}$ studied all patients with suspected lung cancer with no palpable nodes and identified a malignancy in 17 of 55 patients (31\%); however, $16(46 \%)$ of the 35 patients with mediastinal lymphadenopathy had malignant supraclavicular lymph nodes, which is comparable to our series.

Although possible, we have not performed the ultrasound immediately after the CT scan but preferred to do it after the CT scan results and likely diagnosis have been explained to the patient. In an outpatient setting we have tried to set up a flexible service where the ultrasound is often performed on the same day but immediately after the clinic visit. The verbal FNAC result is normally available on the next working day. This technique has potential time and cost savings by reducing the time from referral to diagnosis and reducing the need for invasive mediastinal staging, percutaneous biopsy, and bronchoscopy. We have not attempted to quantify this formally at this stage. The potential for a reduction in the need for surgical mediastinal staging is important as this is an invasive procedure and thoracic surgeons are scarce in the UK.

The procedure is easily learnt. The two chest radiologists involved in the study had no previous experience of neck lymph node sampling. As demonstration of this, there were only six acellular samples (9.5\%) which compares favourably with previously published series. ${ }^{25}$ In some centres radiologists are in short supply and there is no reason why this technique should be dependent on the radiology department. Neck ultrasound and FNAC could be undertaken by appropriately trained staff from any medical speciality, with the potential benefit that this could be performed in an outpatient clinic as part of a "one stop" process.

Ten patients without known distant metastases had CT N2M0 disease and were upstaged to N3M0 by the technique. The prognostic significance of this stage shift is not known. It is likely that many historical studies have similar patients incorrectly staged. Thirty three patients $(32.7 \%)$ in the study had metastatic disease at presentation on CT scanning and the technique, if positive, is a safe, relatively non-invasive and rapid method for gaining a cytological diagnosis without the need for bronchoscopy or CT guided biopsy.

To our knowledge this is the largest published series to date in patients with suspected lung cancer and enlarged mediastinal nodes at staging CT. Using the capillary aspiration technique and more select inclusion criteria, we have achieved a higher diagnostic yield. This is a promising technique, providing a safe method of obtaining a cytological diagnosis and nodal staging in patients with suspected lung cancer.

\section{ACKNOWLEDGEMENTS}

The authors thank Dr Roz Stanley and Jason Rivers for their assistance in collecting the data, Dr Arpan Shrivastava for his assistance in performing ultrasound and FNAC, and the radiology department and thoracic oncology group for supporting the work.

\section{Authors' affiliations}

M Kumaran, R E Benamore, R Vaidhyanath, S Muller, C J Richards, M D Peake, J J Entwisle, Glenfield Hospital, University Hospitals of

Leicester NHS Trust, Leicester, UK

\section{REFERENCES}

1 Jemal A, Thomas A, Mung T, et al. Cancer statistics 2002. CA Cancer J Cin 2002;52:23-47.

2 Anon. National cancer database commission on cancer of the American College of Surgeons and the American Cancer Society. In: Greene FL, Page BL, Fleming ID, et al, eds. American Joint Committee Cancer Staging Handbook. TNM Classification of Malignant Tumours. 6th edn. New York: Springer Verlag, 2002:194.

3 Mountain CF. Regional lymph node classification for lung cancer staging Chest 1997;111:1718-23.

4 Steel BL, Schwartz MR, Ramzy I. Fine needle aspiration biopsy in the diagnosis of lymphadenopathy in 1103 patients. Role, limitations and analysis of diagnostic pitfalls. Acta Cytol 1995;39:76-81.

5 Miller WE, Taylor AM. Biopsy of scalene and supraclavicular lymph nodes: value in diagnosis. Cleveland Clin Q 1965;32:205-9.

6 Monso E, Montserrat JM, Abad J, et al. Usefulness of supraclavicular ultrasonography in the staging of lung cancer. Lung 1992;170:243-4.

7 Luo D, Shi M, Xu Z. Cervical lymph node metastasis: CT, ultrasound versus physical palpation. Zhonghua-Zhong-Liv-Za-Zhi 1998;20:48-50.

8 Takashima S, Sone S, Nomura N, et al. Nonpalpable lymph nodes of the neck: assessment with US and US-guided fine-needle aspiration biopsy. J Clin Ultrasound 1997;25:283-92.

9 Van den Brekel M, Stel HV, Castelijns JA, et al. Lymph node staging in patients with clinically negative examinations by ultrasound and ultrasound guided aspiration cytology. Am J Surg $1991 ; 162: 362-6$.

10 Sugama Y, Kitamura S. Ultrasonographic evaluation of neck and supraclavicular lymph nodes metastasised from lung cancer. Intern Med 1992;31:160-4.

11 Wu CH, Chang YL, Hsu WC, et al. Usefulness of Doppler spectral analysis and power Doppler sonography in the differentiation of cervical lymphadenopathy. Am J Roentgenol 1998;171:503-9.

12 Ahuja AT, Ying M, Ho SS, et al. Distribution of intranodal vessels in differentiating benign from metastatic neck nodes. Clin Radiol 2001;56:197-201

13 Adibelli ZH, Unal G, Gul E, et al. Differentiation of benign and malignant cervical lymph nodes: value of B-mode and color Doppler sonography. Eur J Radiol 1998;28:230-4. 
14 Fultz PJ, Feins RH, Strang JG, et al. Detection and diagnosis of nonpalpable supraclavicular lymph nodes in lung cancer at CT and US. Radiology 2002;222:245-51.

15 van Overhagen H, Brakel K, Heijenbrok MW, et al. Metastases in supraclavicular lymph nodes in lung cancer: assessment with palpation, US and CT. Radiology 2004;232:75-80.

16 Glazer GM, Gross BH, Quint LE, et al. Normal mediastinal lymph nodes: number and size according to American Thoracic Society mapping. Am J Roentgenol 1985;144:261-5.

17 McLoud TC, Bourgouin PM, Greenberg RW, et al. Bronchogenic carcinoma: analysis of staging in the mediastinum with CT by correlative lymph node mapping and sampling. Radiology 1992;182:319-23.

18 Gould MK, Kuschner WG, Rydzak CE, et al. Test performance of positron emission tomography and computed tomography for mediastinal staging in patients with non-small-cell lung cancer: a meta-analysis. Ann Intern Med 2003; 139:879-92.

19 Manhire AR, Charig M, Clelland C, et al. Guidelines for radiologically guided lung biopsy. Thorax 2003;58:920-36.
20 Schatzlein MH, McAuliffe S, Orringer MB, et al. Scalene node biopsy in pulmonary carcinoma: When is it indicated? Ann Thorac Surg 1981;31:322-4.

21 Cozens NJ, Berman L. Fine needle aspiration or core biopsy? In: Ahuja AT, Evans RM, eds. Practical head and neck ultrasound. Greenwich: Medical Media Ltd, 2000:129-44.

22 Hsu JW, Chiang CD, His WH, et al. Superior vena cava syndrome in lung cancer: an analysis of 54 cases. Gaoxing Yi Xue Ke Xue Za Zhi 1995; 11:568-73.

23 Eisele DW, Sherman ME, Koch WM, et al. Utility of immediate on-site cytopathological procurement and evaluation in fine needle aspiration biopsy of head and neck masses. Laryngoscope 1992;102:1328-30.

24 Bearcroft PW, Berman LH, Grant J. The use of ultrasound-guided cuttingneedle biopsy in the neck. Clin Radiol 1995;50:690-5

25 Gupta RK, Naran S, Lallu S, et al. The diagnostic value of fine needle aspiration cytology (FNAC) in the assessment of palpable supraclavicular lymph nodes: a study of 218 cases. Cytopathology 2003;14:201-7.

\section{LUNG ALERT}

Benefits of early pulmonary rehabilitation following acute exacerbation of COPD

$\Delta$ Man W D-C, Polkey Ml, Donaldson N, et al. Community pulmonary rehabilitation after hospitalisation for acute exacerbations of chronic obstructive pulmonary disease: randomised controlled study. BMJ 2004;329:1209-11

$\mathrm{P}$ ulmonary rehabilitation (PR) results in significant improvements to quality of life and both functional and maximum exercise capacity in patients with stable COPD. In this study the benefits of an early outpatient PR programme were compared with usual care at 3 months following hospital admission for acute exacerbation of COPD.

42 patients with acute exacerbation of COPD admitted to hospital were recruited into the study. All the patients received standard treatment including bronchodilators, oral steroids, antibiotics, oxygen, and (if required) non-invasive ventilation. At discharge, patients were randomised to either an early PR programme or usual care, with 21 patients in each group. Baseline assessments 24 hours before discharge were made by measuring exercise capacity with an incremental shuttle walk test. The St George's Respiratory Questionnaire (SGRQ) and Chronic Respiratory Disease Questionnaire (CRQ) were used to measure disease specific health status, with the SF-36 questionnaire employed for generic health status. The PR programme was run by a multidisciplinary team and consisted of twice weekly classes for 8 weeks, each session lasting 2 hours with 1 hour of exercise and 1 hour of educational activity.

After 3 months the results showed significant improvements in exercise capacity, SGRQ, $\mathrm{CRQ}$, and generic health status in the PR group, with fewer visits to A\&E and a trend to a reduced hospital readmission rate. Possible mechanisms to explain the effects of PR include improvements in skeletal muscle function, reduced sensitivity to breathlessness, and life style changes. Early PR thus significantly improves functional capacity and quality of life after discharge following acute exacerbation of COPD.

A Jain
Pinderfields General Hospital, Wakefield, West Yorkshire, UK;
aj1974@rediffmail.com

